\title{
Bioequivalence Studies of A Generic Formulation (SW651K) to the Brand Drug S-1 in Tumor-Bearing Rat Models
}

\author{
Tomoyuki Okabe $^{1 *}$, Takeharu Ogura ${ }^{1}$, Takashi Yoshimura ${ }^{1}$, Yoshiyuki Tanaka ${ }^{1}$, Hiromu Toyoda ${ }^{1}$, Ken-ichi Fujita ${ }^{2}$ and Yasutsuna Sasaki ${ }^{3}$ \\ ${ }^{1}$ Sawai Pharmaceutical Co., Ltd., Biological Research Department, 2-30 Miyahara 5-chome, Yodogawa-ku, Osaka, Japan \\ ${ }^{2}$ Institute of Molecular Oncology, Showa University, 1-5-8 Hatanodai, Shinagawa-ku, Tokyo, Japan \\ ${ }^{3}$ Division of Medical Oncology, Department of Internal Medicine, Showa University School of Medicine, 1-5-8 Hatanodai, Shinagawa-ku, Tokyo, Japan
}

\begin{abstract}
SW651K, a fixed combination of tegafur (FT), 5-chloro-2,4-dihydroxypyridine (CDHP) and potassium oxonate (Oxo), is a generic preparation of $\mathrm{S}-1$, which is widely used to treat gastric cancer in Japan. There is little detailed information of pharmacological effects of SW651K in clinical therapy. However it is not easy to evaluate the PK/PD in clinical trial. Therefore this study examined the bioequivalence of SW651K to S-1 in terms of pharmacokinetics and tumor shrinkage in tumor-bearing rats. Bioequivalence of SW651K to S-1 was first evaluated in Yoshida sarcomabearing rats. Concentrations of FT, 5-fluorouracil (5-FU, the active metabolite of FT), CDHP, and Oxo in plasma, tumor, small intestine, and large intestine were analyzed after a single dose of SW651K. Tumor size was measured during treatment with each formulation for 7 consecutive days. Next, tumor size was measured in human gastric cancer cell (NUGC4)-bearing rats treated for 14 days. Tumor 5-FU concentrations were also analyzed. Tumor size in NUGC4-bearing rats treated with SW651K or S-1 plus cisplatin was also evaluated. SW651K was bioequivalent to S-1 in terms of the pharmacokinetics of all components and of 5-FU in rats. Both formulations also had equivalent antitumor activities in Yoshida sarcoma- and NUGC4 tumor-bearing rats that received monotherapy. Moreover, combined treatment with cisplatin equally potentiated the antitumor effects of both formulations, without increasing body-weight loss in NUGC4 tumor-bearing rats. In conclusion, bioequivalence of SW651K and S-1 was confirmed in terms of pharmacokinetics and antitumor effectiveness in tumor-bearing rats. Our results suggest that SW651K is clinically equivalent to $\mathrm{S}-1$.
\end{abstract}

Keywords: SW651K (ESUEEWAN); S-1 (TS-1); Bioequivalence; Pharmacodynamics; Pharmacokinetics

\section{Introduction}

The generic anticancer drugs are developed in the field of anticancer therapy and are commercially available. Generally, the safety and efficacy of a brand anticancer drug are determined by phase 1,2 and 3 clinical trials. In contrast, clinical trials are not necessarily required by guidelines for the development of generic drugs and are usually not performed. Pharmacokinetic equivalence is considered particularly important for cytotoxic anticancer drugs because of the narrow therapeutic window. Differences in the content and purity of active and inactive pharmaceutical ingredients might have an important impact on pharmacokinetics of the generic cytotoxic anticancer drug and cause the difference in the pharmacodynamics. However the bioequivalent studies of PK/PD using the tumor-bearing rat models had been hardly reported before.

SW651K (ESUEEWAN", Sawai Pharmaceutical Co.) is a generic formulation of oral anticancer drug S-1 (TS-1), developed by Taiho Pharmaceutical Co., similarly consisting of tegafur (FT), 5-chloro-2,4diydropyridine (CDHP), and potassium oxonate (Oxo). Concomitant administration of these formulations and cisplatin is recommended as a first-line treatment for gastric cancer in Japanese therapeutic guidelines [1]. SW651K shows the dissolution pattern similar to S-1 in vitro. FT is a prodrug of the cytotoxic 5-fluorouracil (5-FU), which is gradually converted into 5 -FU mainly by CYP $2 \mathrm{~A} 6$ in the liver. Subsequently, 5-FU is phosphorylated to 5-fluoro-2'deoxyuridine5'-monophosphate (FdUMP), which is an active metabolite of 5-FU that exerts antitumor activity by forming a covalent ternary complex with thymidylate synthase and reduced folate [2]. CDHP increases the blood and intratumoral concentrations of 5-FU by reversibly inhibiting dihydropyrimidine dehydrogenase (DPD), which is the rate-limiting enzyme in the detoxification pathway of 5-FU, and thereby reduces the production of $\alpha$-fluoro- $\beta$-alanine (FBAL), which is known to induce neurotoxicity [3-7]. Oxo reversibly inhibits orotate phosphoribosyltransferase (OPRT), which participates in the phosphorylation of 5-FU in the digestive tract, and thereby decreases gastrointestinal toxicity induced by phosphorylated 5-FU derivatives [4,6-9].

To confirm bioequivalence of SW651K to S-1 in detail, we evaluated the pharmacokinetic as well as pharmacodynamic equivalence of these bland new and generic formulations with the use of tumor-bearing rat models. The pharmacokinetics of FT, active 5-FU, CDHP, and Oxo in plasma, tumor, small intestine, and large intestine were examined in rats given SW651K or S-1. Pharmacological effects, such as antitumor activity and toxicity, were also compared between SW651K and S-1 with or without concurrent treatment with cisplatin.

\section{Materials and Methods \\ Drugs}

The test formulation SW651K (Sawai Pharmaceutical Co., Osaka,

*Corresponding author: Tomoyuki Okabe, Sawai Pharmaceutical Co., Ltd., Biological Research Department, Tel: +81-6-6105-5731; Fax: +81-6-6394-7318 E-mail: t.okabe@sawai.co.jp

Received February 26, 2016; Accepted March 04, 2016; Published March 11 2016

Citation: Okabe T, Ogura T, Yoshimura T, Tanaka Y, Toyoda H, et al. (2016) Bioequivalence Studies of A Generic Formulation (SW651K) to the Brand Drug S-1 in Tumor-Bearing Rat Models. J Bioequiv Availab 8: 112-117. doi:10.4172/ jbb. 1000279

Copyright: (C) 2016 Okabe T, et al. This is an open-access article distributed under the terms of the Creative Commons Attribution License, which permits unrestricted use, distribution, and reproduction in any medium, provided the original author and source are credited. 
Japan) and the reference formulation S-1 (Taiho Pharmaceutical Co., Tokyo, Japan) were used in this study. Each formulation was administered after being suspended in $0.5 \%$ hypromellose solution to achieve the designated concentration. The doses of these drugs are expressed as the FT dose. Cisplatin (Sigma-Aldrich, St. Louis, MO, USA) was used after being dissolved and diluted with saline.

\section{Animals}

Four-week-old male Donryu rats and male F344 / NJcl-rnu / rnu nude rats were purchased from Japan SLC Inc. (Shizuoka, Japan) and Clea Japan Inc. (Tokyo, Japan), respectively. The animals were used in the studies after preliminary rearing for 1 week. All animal protocols were approved by the Animal Care Committee in Sawai Pharmaceutical Co. (Osaka, Japan) and performed according to institutional guidelines. The rat were maintained under a controlled environment $\left(20-26^{\circ} \mathrm{C}\right.$, $55 \%$ relative humidity, $12 \mathrm{~h}$ light-dark cycle) with free access to food and water.

\section{Tumor-bearing rat model}

Yoshida sarcoma (YS-TC) cells, which are derived from ascites tumors in rats, were supplied by Cell Resource Center for Biomedical Research, Institute of Development, Aging, and Cancer, Tohoku University (Sendai, Japan). The Yoshida sarcoma-bearing animal models were established by subcutaneous injection of this cell suspension $\left(5 \times 10^{5}\right.$ cells $)$ into the right side of the abdomen of Donryu rats. NUGC4 cells, which are a human gastric cancer strain, were obtained from the Riken BioResource Center (Ibaragi, Japan). The NUGC4 tumor-bearing animal models were created by implantation of tumor fragments approximately $3 \mathrm{~mm}$ diameter into the right side of the abdomen of nude rats.

\section{Measurement of antitumor activity in the Yoshida sarcoma- and the NUGC4 tumor-bearing rat model}

The animals were orally given SW651K or S-1 at each dose per day, according to Figure 1. To examine the antitumor effects of SW651K or $\mathrm{S}-1$ in combination with cisplatin, $5 \mathrm{mg} / \mathrm{kg}$ of cisplatin was injected into the caudal vein one time on the day after dividing the rats. The length of the major and minor axes of the tumor were measured, and tumor volume was calculated daily or once every 2 days by the following formula: Estimated tumor volume $\left(\mathrm{mm}^{3}\right)=($ major axis, $\mathrm{mm} \times$ minor axis, $\mathrm{mm}^{2}$ ) / 2. The results of each group were evaluated on the basis of the inhibition ratio of tumor growth $(\mathrm{T} / \mathrm{C}[\%]=$ average estimated tumor volume of a drug administration group / average estimated tumor volume of the control group $\times 100$ ), calculated according to the formula of the screening method [10]. Results in which T / C was $42 \%$ or less were deemed "valid."

\section{Assessment of pharmacokinetics in the tumor-bearing rat model}

The Yoshida sarcoma-bearing rats were orally given SW651K or S-1 at a dose of $15 \mathrm{mg} / \mathrm{kg}$ on day 8 after implantation. Blood was collected from the abdominal aorta $0.51,2,4,6,10$, and $24 \mathrm{~h}$ after drug administration. Then, the rats were sacrificed by exsanguination, and the tumor tissues, small intestines, and large intestines were collected. To homogenize the tissue samples, a homogenizing solution $(100 \mathrm{mM}$ ammonium formate $[\mathrm{pH} 8.6]$ containing $5 \%[\mathrm{v} / \mathrm{v}]$ dibutylamine) was added in an amount equivalent to 2 times and 5 times the weight of the tumor and intestinal tissues, respectively. The tissue homogenate was centrifuged, and a supernatant was obtained. The plasma was diluted with twice the amount of the homogenizing solution, and all samples
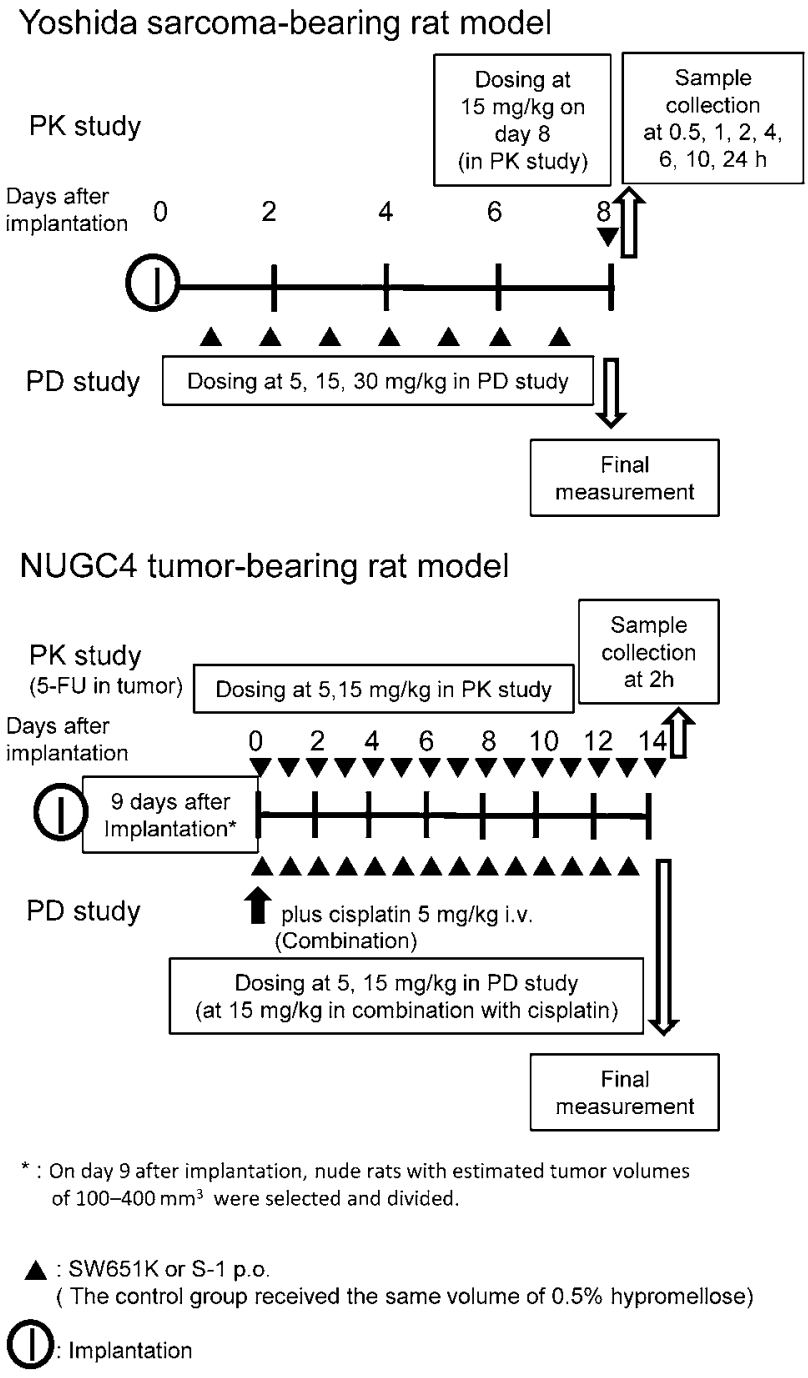

Figure 1: Scheme of pharmacokinetic and pharmacodynamics studies.

were ultrafiltrated (Amicon Ultra-0.5 3KDa; Merck Millipore, Billerica, MA, USA). Subsequently, the processed samples were examined using LC/MS. In comparison of the 5-FU concentrations in the NUGC4tumor during continuous administration, the 5-FU concentration was measured according to the modified method of Hara et al. [11]. An internal standard (5-bromouracil) solution and a homogenizing solution ( $0.5 \mathrm{M}$ sodium dihydrogen phosphate) at amounts equivalent to the weight of the specimens collected were added to homogenize the samples. Ethyl acetate was added to the homogenate solution, and the extracted liquid was evaporated to dryness and dissolved in distilled water. Then, the samples were analyzed by UV detection $(265 \mathrm{~nm})$.

\section{Bioequivalence}

Calculations of pharmacokinetic variables and comparison between groups after confirming the homoscedasticity were performed with EXSUS version 8.0.0 and BESTS version 3.0.4 software (CAC EXICARE, Tokyo Japan). The area under the concentration-time curve from 0 to $24 \mathrm{~h}\left(\mathrm{AUC}_{0-24 \mathrm{~h}}\right)$ after treatment was calculated using the trapezoidal rule with 7 measurement points. Bioequivalence was evaluated on the basis of the $\mathrm{AUC}_{0-24 \mathrm{~h}}$ and $\mathrm{C}_{\max }$ ratio of the SW651K 
group as compared with the corresponding values in the S-1 group, after logarithmic transformation of the variables. Both formulations were deemed equivalent when the difference ratios of the $\mathrm{AUC}_{0-24 \mathrm{~h}}$ and $\mathrm{C}_{\max }$ were within the range of 0.9-1.11.

\section{Results}

Pharmacokinetic profiles of 5-FU, FT, CDHP, and Oxo in plasma

Both SW651K and S-1 formulations showed similar time courses of plasma concentrations of 5-FU, FT, CDHP, and Oxo (Figure 2a). The bioequivalence of SW651K and S-1 was evaluated on the basis of differences between the two formulations in the $\mathrm{AUC}_{0-24 \mathrm{~h}}$ and $\mathrm{C}_{\max }$ values for 5-FU, FT, CDHP, and Oxo. The ratios of the $\mathrm{AUC}_{0-24 \mathrm{~h}}$ and $\mathrm{C}_{\max }$ for 5-FU, FT, CDHP, and Oxo in the SW651K group to the respective values in the S-1 group were in the ranges of 0.93-0.99 and 0.93-1.04, respectively. Therefore, the bioequivalence in pharmacokinetics of concentrations 5-FU, FT, CDHP, and Oxo in plasma between SW651K and S-1 was confirmed.

\section{Pharmacokinetic profiles of 5-FU, FT, CDHP, and Oxo in} tumor and intestinal tissues of Yoshida sarcoma-bearing rats

The pharmacokinetic profiles of 5-FU, FT, CDHP, and Oxo in tumor, small and large intestinal tissues for SW651K were similar to those for $\mathrm{S}-1$ (Figures 2b-2d). The ratios of the $\mathrm{AUC}_{0-24 \mathrm{~h}}$ and $\mathrm{C}_{\max }$ for active 5-FU in tumor tissue in the SW651K group to the respective values in the S-1 group ranged from 0.98 to 1.10 . The higher concentrations of Oxo in small and large intestine than those in plasma or tumor were observed in both formulations. The $\mathrm{AUC}_{0-24 \mathrm{~h}}$ of Oxo in intestinal tissues was more than about 16 and 37 times higher than those in plasma and tumor in both formulations. These results indicate that components and the active 5-FU in SW651K were similarly distributed in the tumor and intestinal tissues to $\mathrm{S}-1$ in the rats.

\section{Antitumor activity of SW651K and S-1 in Yoshida sarcoma- and NUGC4 tumor-bearing rats}

In the Yoshida sarcoma-bearing rat model, the antitumor effects of the SW651K and S-1 did not differ significantly at any doses and increased in a dose-dependent manner from $5 \mathrm{mg} / \mathrm{kg}$ (Figure 3a). The $\mathrm{T} / \mathrm{C}(\%)$ values in the rats given SW651K and S- 1 at a dose of $15 \mathrm{mg} / \mathrm{kg}$ were $24.7 \%$ and $23.8 \%$ respectively, indicating that both formulations were deemed to be valid.

In a human gastric tumor-bearing rat model, the SW651K and S-1 demonstrated significant antitumor effects at a dose of $15 \mathrm{mg} / \mathrm{kg}$ on day 14 as compared with the control group. There were no significant differences in the antitumor effects between the two formulations at any doses. Since, T/C (\%) at a dose of $15 \mathrm{mg} / \mathrm{kg}$ was $20.9 \%$ in the SW651K group and $21.5 \%$ in the S-1, both formulations at a dose of $15 \mathrm{mg} / \mathrm{kg}$ were deemed to be valid as compared with the control group. After the final measurement of tumor size, the rats were given a same dose ( 5 and
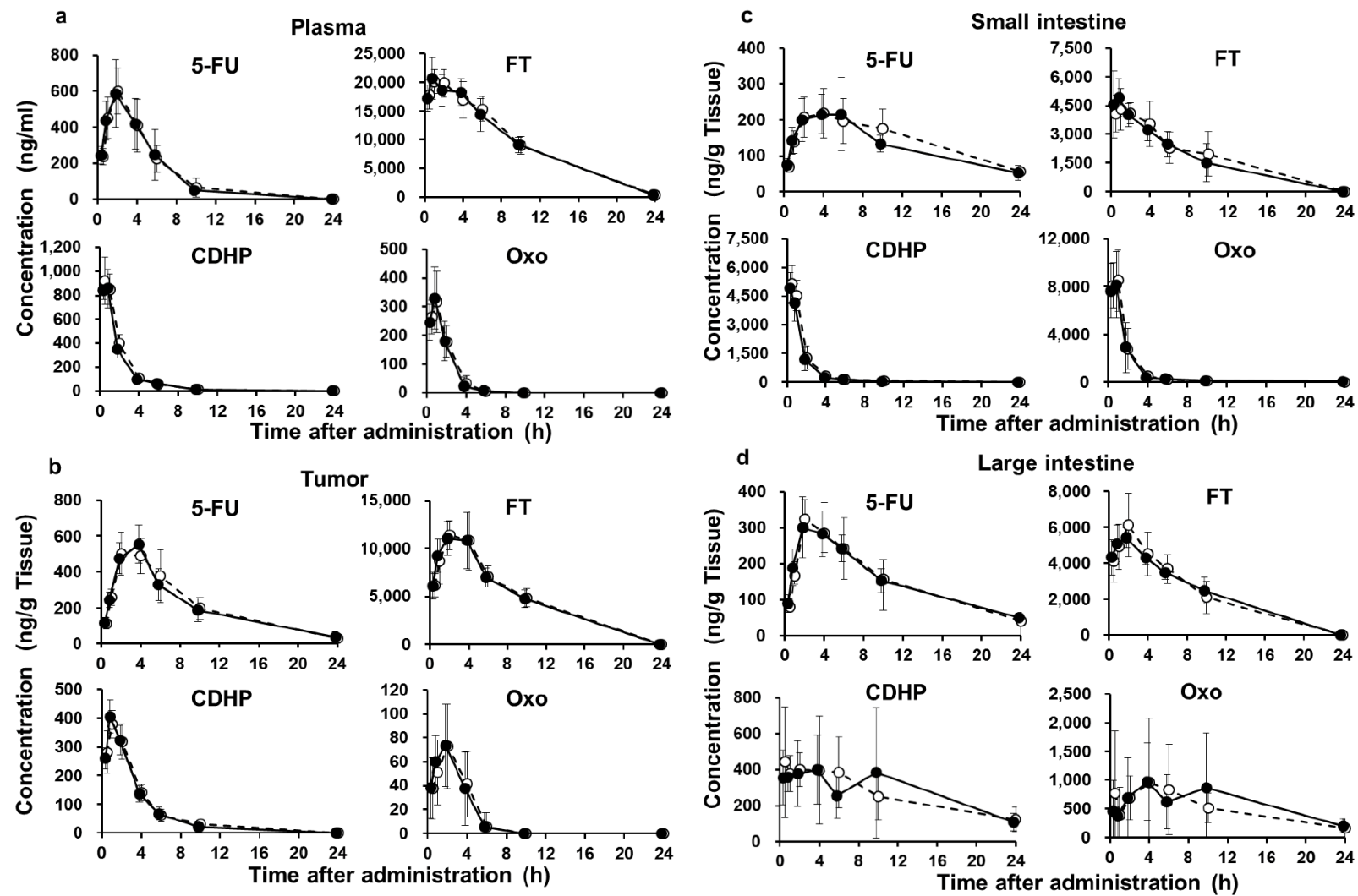

Figure 2: Pharmacokinetic profiles of 5-FU, FT, CDHP, and Oxo in plasma (a): tumor (b): small intestine (c): and large intestine (d): after administration of SW651K and $\mathrm{S}-1$.

SW651K $15 \mathrm{mg} / \mathrm{kg}$ (closed triangles) and S-1 $15 \mathrm{mg} / \mathrm{kg}$ (open triangles) were orally administered once on day 8 after implantation of Yoshida sarcoma-bearing rats Values are means \pm standard deviation in 9 rats. 
Citation: Okabe T, Ogura T, Yoshimura T, Tanaka Y, Toyoda H, et al. (2016) Bioequivalence Studies of A Generic Formulation (SW651K) to the Brand Drug S-1 in Tumor-Bearing Rat Models. J Bioequiv Availab 8: 112-117. doi:10.4172/jbb.1000279

$15 \mathrm{mg} / \mathrm{kg}$ ) of SW651K and S-1 on day 15 . The $5-\mathrm{FU}$ concentrations in tumor tissue were measured at $2 \mathrm{~h}$ after the final treatment. The 5-FU concentrations in tumor were $150.5 \pm 22.3$ and $759.2 \pm 93.3 \mathrm{ng} / \mathrm{g}$ for rats given 5 and $15 \mathrm{mg} / \mathrm{kg}$ of SW651K, respectively, and $148.9 \pm 18.7$ and $752.8 \pm 87.9 \mathrm{ng} / \mathrm{g}$ for rats given the same doses of S-1. These results indicate that both formulations have equivalent antitumor activities in Yoshida sarcoma- and human gastric tumor-bearing rat model.

\section{Toxicity in Yoshida sarcoma-and NUGC4 tumor-bearing rats}

In the Yoshida sarcoma-bearing rats given SW651K or S-1, body weights significantly and dose-dependently decreased at doses of 5 $\mathrm{mg} / \mathrm{kg}$ or higher as compared with control (Figure $3 \mathrm{~b}$ ). There were no significant differences in body weight between the formulations at any doses on day 8 . The patterns of body weight change in human gastric tumor-bearing rats were similar to those in Yoshida sarcoma-bearing rats (Figures $4 \mathrm{a}$ and $4 \mathrm{~b}$ ). These results indicate that both formulations have equivalent toxicities in Yoshida sarcoma- and human gastric tumor-bearing rats.

Comparison of the pharmacological effects of SW651K and S-1 in combination with cisplatin in NUGC4 tumor-bearing rats

Tumor growth was significantly suppressed in all SW651K and
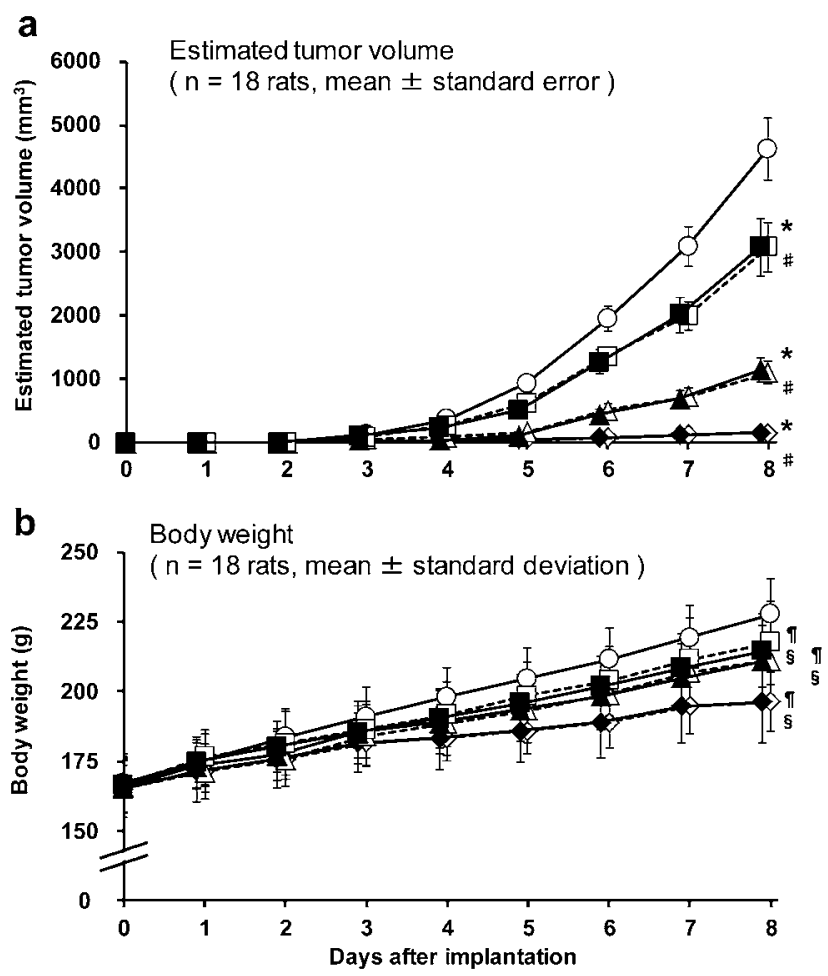

Figure 3: Pharmacological effects of SW651K and S-1 on estimated tumor volume (a) and body weight (b) in Yoshida sarcoma-bearing rats.

Vehicle (control, open circles), SW651K (5 mg/kg, closed squares; $15 \mathrm{mg} /$ $\mathrm{kg}$, closed triangles; $30 \mathrm{mg} / \mathrm{kg}$, closed diamonds), and S-1 (5 mg/kg, open squares; $15 \mathrm{mg} / \mathrm{kg}$, open triangles; $30 \mathrm{mg} / \mathrm{kg}$, open diamonds) were orally administered once a day on the day after Yoshida sarcoma implantation for 7 consecutive days.

\#: $p<0.025$ SW651K vs. Control, *: $p<0.025$ S-1 vs. Control (ShirleyWilliams' multiple comparison tests, 1 -sided)

$\S: p<0.025$ SW651K vs. Control, $\mathbb{\eta}: p<0.025$ S-1 vs. Control (Williams' multiple comparison tests, 1 -sided)

There were no significant differences in the pharmacological effects between the two formulations at any doses.
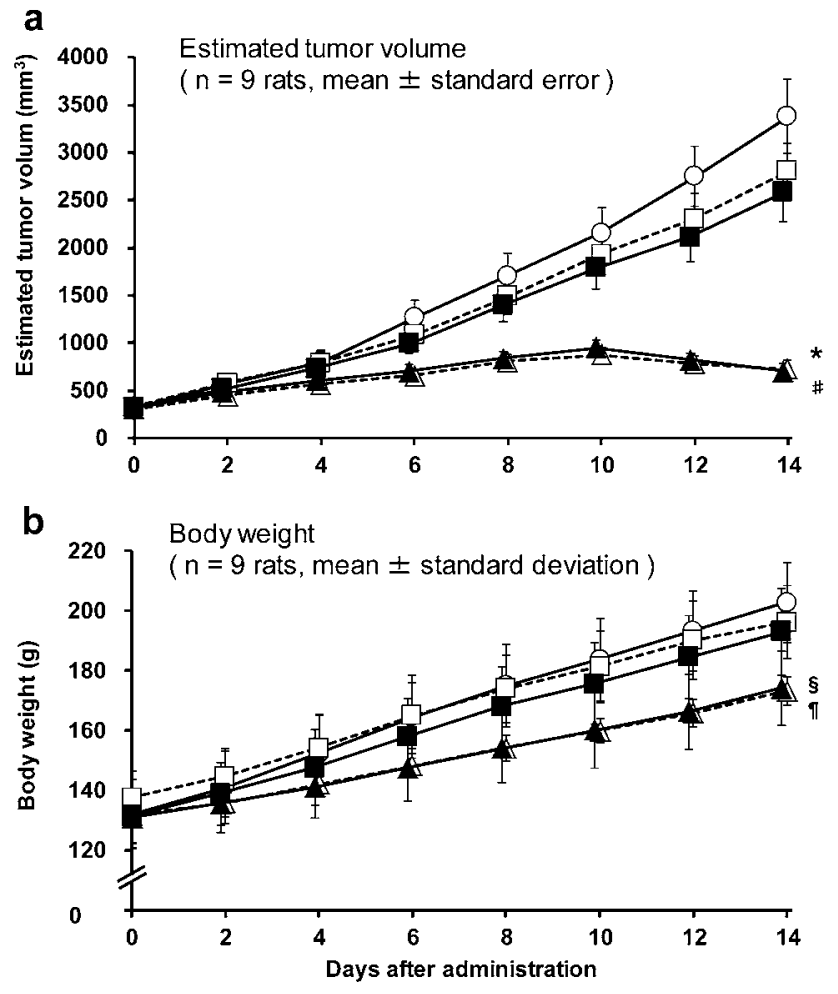

Figure 4: Pharmacological effects on estimated tumor volume (a) and body weight (b) in NUGC4 tumor-bearing rats given SW651K or S-1.

Vehicle (control, open circles), SW651K (5 mg/kg, closed squares; $15 \mathrm{mg} / \mathrm{kg}$, closed triangles), and S-1 ( $5 \mathrm{mg} / \mathrm{kg}$, open squares; $15 \mathrm{mg} / \mathrm{kg}$, open triangles) were orally administered once daily on the day 10 after implantation for 14 consecutive days.

$\#: p<0.05$ SW651K vs. Control, *: $p<0.05$ S-1 vs. Control (Steel multiple comparison tests, 2-sided)

$\S: p<0.05$ SW651K vs. Control, $\uparrow: p<0.05$ S-1 vs. Control (Dunnett's multiple comparison tests, 2-sided)

There were no significant differences in the pharmacological effects between the two formulations at any doses.

cisplatin or S-1 and cisplatin treated groups, except cisplatin alone (Figure 5a). The cisplatin combination groups showed significantly higher antitumor activity than groups receiving SW651K or S-1 alone. Addition of cisplatin decreased the T/C (\%) from $21.8 \%$ to $10.4 \%$ in the SW651K group and from $24.0 \%$ to $11.8 \%$ in the S-1 group, whereas further reduction in body weight was not observed in the groups received additional cisplatin (Figure $5 b$ ). There were no significant differences in antitumor activity or body weight loss between the SW651K plus cisplatin group and the S-1 plus cisplatin group.

\section{Discussion}

In Japan, national healthcare costs have been progressively rising and accounting for a higher proportion of the national budget, and drug-related costs account for a considerable proportion of the national healthcare expenditure. In general, the increased use of generic drugs is expected to reduce healthcare costs. In particular, patients who receive high-priced drugs such as anticancer chemotherapy are expected to substantially benefit from the increased use of generic drugs, contributing to a lower financial burden on the patient. Lower drugrelated costs will allow patients to receive the high-quality medical care by additionally implementing optional therapy. However, the current 

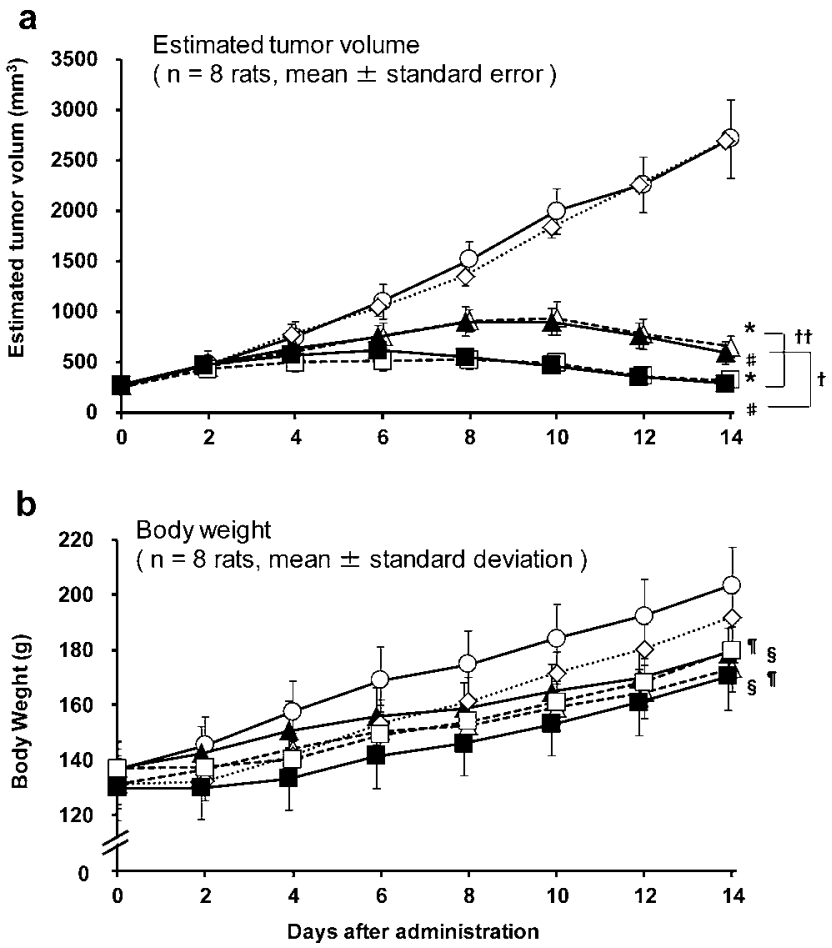

Figure 5: Pharmacological effects on estimated tumor volume (a) and body weight (b) in NUGC4 tumor-bearing rats given SW651K plus cisplatin or S-1 plus cisplatin.

Vehicle (control, open circles), SW651K (15 mg/kg, closed triangles), and $\mathrm{S}-1$ (15 $\mathrm{mg} / \mathrm{kg}$, open triangles). On the day after grouping, a single dose of cisplatin $5 \mathrm{mg} / \mathrm{kg}$ was injected into the caudal vein of rats in the cisplatin (open diamonds), SW651K plus cisplatin (closed squares), and S-1 plus cisplatin groups (open squares). After the injection of cisplatin, SW651K or S-1 was administered to the rats in the SW651K plus cisplatin and S-1 plus cisplatin groups and continued for 14 days.

\#: $p<0.05$ SW651K vs. Control, *: $p<0.05$ S-1 vs. Control (Steel multiple comparison tests, 2 -sided), $\S: p<0.05$ SW651K vs. Control, $\mathbb{T}: p<0.05 \mathrm{~S}-1 \mathrm{vs.}$ Control (Dunnett's multiple comparison tests, 2-sided), $\uparrow: p<0.05$ SW651K vs. plus cisplatin, $\uparrow \uparrow: p<0.05 \mathrm{~S}-1$ vs. plus cisplatin ( $t$ - tests, 2-sided). There were no significant differences in the pharmacological effects between the two formulations with or without cisplatin.

rate of generic drug use is far lower in Japan than in Europe and the United States. Concern about the safety and effectiveness of generic drugs may be one reason for the low usage rate in Japan. Therefore we thought that it was necessary to confirm the pharmacokinetics and pharmacodynamics profiles in the development of antitumor drug such as S-1 that had the narrow therapeutic window and the interaction by the combined components.

In the present study, we confirmed the equivalence in plasma concentrations of 5-FU between Yoshida sarcoma-bearing rats given SW651K or S-1 (Figure 2a). The similar pharmacokinetic profile of active 5-FU in plasma must be related to that in tumor (Figure $2 \mathrm{~b}$ ), and thus resulting in the similar antitumor effects of these two formulations in Yoshida sarcoma-bearing rats (Figure 3a). Plasma concentrations of 5-FU and intestinal concentrations of Oxo are considered to be associated with toxic effects of SW651K and S-1. The equivalence of these concentrations might be related to the similar body weight loss seen in these two groups of rats (Figure $3 b$ ). One of possible reasons for the pharmacokinetic equivalence might be the quite similar dissolution rate of all components in SW651K formulation to that in S-1. Thus, we conclude that generic drug SW651K is equivalent to the brand drug S-1 in terms of pharmacokinetics and pharmacodynamics in Yoshida sarcoma-bearing rat models.

In Japan, S-1 is extensively used for the treatment of patients with advanced gastric cancer as the first-line drugs [1]. Recently, a combination of S-1 and cisplatin has been recommended as standard treatment for advanced gastric cancer on the basis of the results of clinical studies such as the SPIRITS trial [12]. S-1 is also approved for the use in adjuvant chemotherapy in gastric cancer patients who underwent gastrectomy [1]. Cisplatin is thought to increase the inhibition of thymidylate synthase by elevating the amount of reduced folate in tumor cells $[13,14]$. Combination with SW651K or S-1, and cisplatin enhanced the antitumor activity without greater body weight loss (Figure $5 \mathrm{~b}$ ). Therefore, concomitant treatment with cisplatin is thought to be very useful from the viewpoints of therapeutic effectiveness and side effects. The similar antitumor effects and body weight loss in NUGC4 tumor-bearing rats treated with SW651K or S-1 alone (Figure 4), or in combination with cisplatin (Figure 5) suggest that SW651K would be applicable for the treatment of patients with gastric cancer and would be expected to show the same efficacy and toxicity to $\mathrm{S}-1$ in humans. Because the price of SW651K is approximately $40 \%$ lower than that of S- 1 and gastric cancer patients receive S-1 on a longterm basis, the use of the generic formulation must contribute to lower the financial burden on the patient.

On pharmacokinetic analysis, slight differences in the concentrations of CDHP and Oxo in large intestinal tissue between SW651K and S-1 were observed (Figure 2d). Generally, substances with low liposolubility show low absorption efficiency and variable concentrations in the intestinal tract. Variations in pharmacokinetic variables in large intestinal tissue were most likely caused by the low absorption efficiency of CDHP and Oxo in the large intestine, as reported previously [15].

In conclusion, the development of generic formulations of S-1 has great social and economic implications. And demonstration of the bioequivalence of generic drugs to brand drugs in terms of pharmacokinetics and pharmacodynamics is expected to help clinicians decide whether to switch from a brand drug to a generic drug. In this study, we confirmed the bioequivalence of the oral anticancer drugs SW651K and S-1 by comparing their pharmacokinetics, antitumor activity and toxicity in a Yoshida sarcoma- and human gastric cancerbearing rat model, thus $\mathrm{SW} 651 \mathrm{~K}$ is clinically available as S-1 for anticancer therapy and the use of SW651K contributes to a lower financial burden on the patient.

\section{Conflict of Interest}

Tomoyuki Okabe, Takeharu Ogura, Takashi Yoshimura, Yoshiyuki Tanaka and Hiromu Toyoda are employees of Sawai Pharmaceutical Co., Ltd. For the remaining authors there are no conflicts of interest.

\section{References}

1. Japanese Gastric Cancer Association (2011) Japanese gastric cancer treatment guidelines 2010 (ver. 3). Gastric Cancer 14: 113-123.

2. Ichikawa W (2006) Prediction of clinical outcome of fluoropyrimidine-based chemotherapy for gastric cancer patients, in terms of the 5 -fluorouracil metabolic pathway. Gastric Cancer 9: 145-155.

3. Fukushima M, Satake $H$, Uchida J, Shimamoto $Y$, Kato T, et al. (1998) Preclinical antitumor efficacy of S-1: a new oral formulation of 5-fluorouracil on human tumor xenografts. Int J Oncol 13: 693-698.

4. Kato T, Shimamoto $\mathrm{Y}$, Uchida J, Ohshimo H, Abe M, et al. (2001) Possible 
Citation: Okabe T, Ogura T, Yoshimura T, Tanaka Y, Toyoda H, et al. (2016) Bioequivalence Studies of A Generic Formulation (SW651K) to the Brand Drug S-1 in Tumor-Bearing Rat Models. J Bioequiv Availab 8: 112-117. doi:10.4172/jbb.1000279

regulation of 5-fluorouracil-induced neuro- and oral toxicities by two biochemical modulators consisting of S-1, a new oral formulation of 5-fluorouracil. Anticancer Res 21: 1705-1712.

5. Shirasaka T, Nakano K, Takechi T, Satake H, Uchida J, et al. (1996) Antitumor activity of $1 \mathrm{M}$ tegafur-0.4 M 5-chloro-2,4-dihydroxypyridine-1 $\mathrm{M}$ potassium oxonate (S-1) against human colon carcinoma orthotopically implanted into nude rats. Cancer Res 56: 2602-2606.

6. Shirasaka T, Shimamoto Y, Ohshimo H, Yamaguchi M, Kato T, et al. (1996) Development of a novel form of an oral 5-fluorouracil derivative (S-1) directed to the potentiation of the tumor selective cytotoxicity of 5 -fluorouracil by two biochemical modulators. Anticancer Drugs 7: 548-557.

7. Takechi T, Nakano K, Uchida J, Mita A, Toko K, et al. (1997) Antitumor activity and low intestinal toxicity of S-1, a new formulation of oral tegafur, in experimental tumor models in rats. Cancer Chemother Pharmacol 39: 205-211.

8. Shirasaka T, Shimamoto Y, Fukushima M (1993) Inhibition by oxonic acid of gastrointestinal toxicity of 5-fluorouracil without loss of its antitumor activity in rats. Cancer Res 53: 4004-4009.

9. Yoshisue K, Hironaga K, Yamaguchi S, Yamamoto A, Nagayama S, et al. (2000) Reduction of 5-fluorouracil (5-FU) gastrointestinal (GI) toxicity resulting from the protection of thymidylate synthase (TS) in GI tissue by repeated simultaneous administration of potassium oxonate (Oxo) in rats. Cancer Chemother Pharmacol 46: 51-56.
10. Goldin A, Venditti JM, Macdonald JS, Muggia FM, Henney JE, et al. (1981) Current results of the screening program at the Division of Cancer Treatment, National Cancer Institute. Eur J Cancer 17: 129-142.

11. Hara Y, Kono A, Tanaka M (1984) Measurement of 5'-deoxy-5-fluorouridine (5'-DFUR) by high-performance liquid chromatography and studies on pharmacokinetics of 5'-DFUR and 5-fluorouracil by oral and intravenous administration. Jpn J Cancer Chemother 11: 2261-2266.

12. Koizumi W, Narahara H, Hara T, Takagane A, Akiya T, et al. (2008) S-1 plus cisplatin versus S-1 alone for first-line treatment of advanced gastric cancer (SPIRITS trial): a phase III trial. Lancet Oncol 9: 215-221.

13. Araki H, Fukushima M, Kamiyama Y, Shirasaka T (2000) Effect of consecutive lower-dose cisplatin in enhancement of 5-fluorouracil cytotoxicity in experimental tumor cells in vivo. Cancer Lett 160: 185-191.

14. Shirasaka T, Shimamoto Y, Ohshimo H, Saito H, Fukushima M (1993) Metabolic basis of the synergistic antitumor activities of 5-fluorouracil and cisplatin in rodent tumor models in vivo. Cancer Chemother Pharmacol 32: 167-172.

15. Masuda H, Ikeda K, Toko K, Nagayama S, Kawaguchi Y, et al. (1997) Disposition of components of new anti-cancer drug S-1 (1): absorption and excretion of components of S-1 after single administration to rats. Drug Metab Pharmacokinet 12: 289-300. 